\title{
Electron Capture Detector Based on X-rays
}

\author{
Nic Solle, Erik Bunert and Stefan Zimmermann \\ Institute of Electrical Engineering and Measurement Technology, Leibniz University Hannover, \\ Appelstr. 9A, 30167 Hannover, Germany \\ Contact: solle@geml.uni-hannover.de
}

\section{Introduction}

Electron capture detectors (ECDs), first introduced by J.E. Lovelock in 1958 [1], are characterized by their simple design and low limits of detection in the pptv range for substances with high electron affinity such as pesticides or halogenated compounds $[1,2]$. Thus, ECDs are often used in environmental analytics [3]. In classical ECDs a radioactive ${ }^{63} \mathrm{Ni}$ foil is used to ionize a carrier gas, e.g. nitrogen, generating thermalized electrons. These electrons drift towards a detector in an electric field resulting in a constant electron detector current. Analytes with sufficient electron affinity capture the thermalized electrons and are flushed out of the ECD with the carrier gas flow. Thus, the electron current reaching the detector is decreased and a measure for the analyte concentration. Pulsed operation was later introduced by R.J. Maggs et al. which increased the linear dynamic range to $10^{4}$ [4]. Usually, radioactive ionization sources are used in ECDs because they require no maintenance and no additional power supply. However, radioactive materials are subject to regulations and costs for disposal. Therefore, despite the excellent detection limits for substances with high electron affinities, today, radioactive ECDs can be rarely found as detectors, e.g. in gas chromatography (GC).

Existing alternatives to radioactive ionization sources include non-radioactive electron sources based on helium discharge and thermionic emission [3,5-7]. Both methods show comparable results in terms of detection limits and linearity but could not be established, e.g. in GC. Helium discharge ionization sources require additional periphery such as pumps, helium gas and pulsed power supplies, and the available thermionic electron emitters cannot operate at high temperatures needed for most GC applications.

Another approach to replace the radioactive sources in ECDs is to use the ionizing radiation emitted by low energy X-ray sources. Such X-ray sources can be operated with an accelerating voltage of less than $5 \mathrm{kV}$ and are not subject to costly regulatory requirements during operation. However, due to the different radiation characteristic, the ECD reaction region needs to be adapted.

\section{Experimental}

The classical ECD uses a coaxial structure to form the reaction chamber, as presented in Fig. 1. Electrode 1 consists of a ${ }^{63} \mathrm{Ni}$ foil acting as the ionization source and Electrode 2 in the middle as the detector electrode. The carrier gas with the analytes eluting from the GC column flows axially between the detector electrode and the ${ }^{63} \mathrm{Ni}$ foil through the reaction chamber. For an increased gas flow for faster response, or when using helium or hydrogen as carrier gas, nitrogen can be added as make-up gas before entering the ECD.

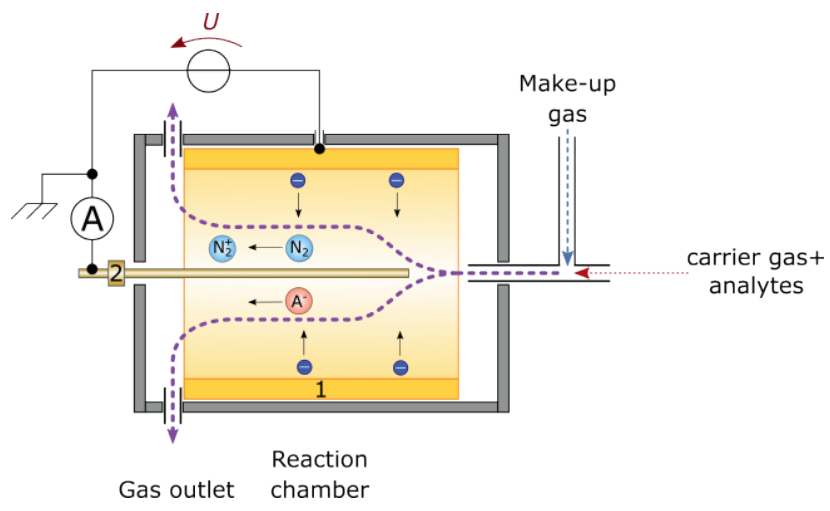

Fig. 1: Reaction chamber of an ECD based on a ${ }^{63} \mathrm{Ni}$ foil (1) with the detector electrode (2).

The radioactive $\beta$ - decay of ${ }^{63} \mathrm{Ni}$ generates high energetic electrons, which ionize the nitrogen molecules. This eventually leads to a high amount of thermalized electrons. By applying an electric field by a negative voltage $U$ pointing from the detector electrode to the ${ }^{63} \mathrm{Ni}$ foil the thermalized electrons are accelerated towards the detector electrode, leading to a constant electron current on the detector. Now, electron affine analytes capture the electrons and are flushed out by the gas flow. Thus, the electron current decreases and the amplitude of the decrease is proportional to the analyte concentration. The voltage $U$ can be pulsed to obtain a higher linearity of the ECD (pulsed operation). Therefore, the measured electron current is fed to a proportional-integral-derivative controller (PID controller). This PID controller fixes the electron current to a set point by applying the negative voltage $U$ with a defined pulse length while changing the pulse frequency. The Frequency 
change is then directly proportional to the analyte concentration $[1,2,4]$.

Alternatively, an X-ray source can be used to ionize the carrier (or make-up) gas via photoionization $[8,9]$ and thus generating free electrons leading to thermalized electrons. While the ${ }^{63} \mathrm{Ni}$ foil emits high energetic electrons from the entire inner surface, the X-ray source just radiates $\mathrm{X}$-rays via a plane outlet window. Considering the radiation characteristic of the used $X$ ray source the reaction chamber of the ECD based on X-rays contains three ring electrodes while the Xray source is attached at one side of the reaction chamber, see Fig. 2.

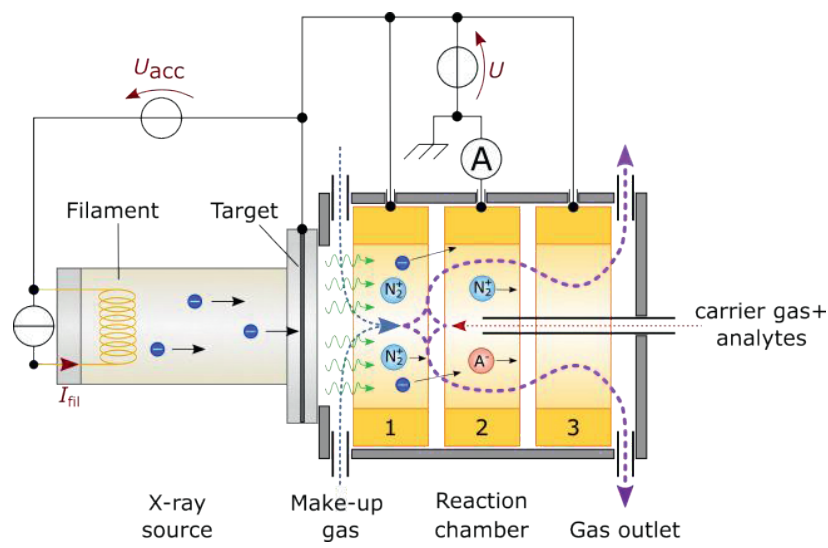

Fig. 2: Reaction chamber of an ECD based on X-rays with three ring electrodes: pusher electrode (ring 1), detector electrode (ring 2) and block electrode (ring 3).

Placed at the center axis, the X-ray source can radiate into the whole reaction chamber. Therefore, the inner diameter of the ring electrodes corresponds to the size of the X-ray outlet window of the source. The carrier gas, here nitrogen, containing the analytes is directed through a capillary reaching into the reaction chamber and enters the reaction chamber at the middle of ring 2. Due to the geometry of the chamber and the opposing make-up gas flow entering at the X-ray source, the analytes are flushed out through the gas outlet. The X-ray source ionizes the carrier gas or the make-up gas respectively and thus the required thermalized electrons are generated. By applying the negative voltage $U$ at the outer rings an electric field pointing from the pusher electrode (ring 1) and the block electrode (ring 3 ) to the detector electrode (ring 2 ) is generated. Thus, the thermalized electrons drift against the analyte gas flow towards the middle of the chamber, as shown in Fig. 2. Here, the electrons can be captured by the analytes, resulting in a decreased electron current at the detector electrode. Since the voltage $U$ is applied at the block electrode the electrons are prevented to be flushed out with the gas flow.

The ionization capability of the X-ray source depends on the energy density of the X-rays. Therefore, the accelerating voltage $U_{\text {acc }}$ and the filament current $I_{\text {fil }}$ can be adjusted to set the energy density. A detailed description of the dependency of the energy density on the acceleration voltage and the filament current can be found in $[8,9]$.

To characterize the ECD using an X-ray source, 1,1,2-Trichloroethane $(1,1,2-$ TCE) was chosen as a model analyte. 1,1,2-TCE was mixed with nitrogen and flushed through the reaction chamber with a flow rate of $5 \mathrm{ml} / \mathrm{min}^{1}$. In addition, nitrogen was used as the make-up gas with a flow of $10 \mathrm{ml} / \mathrm{min}$, which results in a total flow of $15 \mathrm{ml}_{\mathrm{s}} / \mathrm{min}$ through the reaction chamber. For the following experiments, the voltage $U$ was typically set to an amplitude of $-32 \mathrm{~V}$. The electron current was amplified by a transimpedance amplifier [10], developed and adapted at our institute, and read out by a Keysight 34461A multimeter with an averaging time of $200 \mathrm{~ms}$. The temperature of the reaction chamber was $80^{\circ} \mathrm{C}$. The acceleration voltage was set to a constant value of $U_{\text {acc }}=4.9 \mathrm{kV}$ and the filament current to $I_{\text {fil }}=530 \mathrm{~mA}$.

\section{Results and Discussion}

For a first benchmarking, the reached limit of detection and linearity of the ECD based on X-rays for 1,1,2-TCE were compared to classical ECDs. For pulsed operation, the optimum pulse width and amplitude $U$ had to be determined at which most of the electrons reach the detector, but no negative ions. The maximum electron current difference was determined by varying $U$ with and without an analyte in the ECD.

However, the first measurements showed a positive detector current, which cannot be caused by electrons or negative ions. To investigate this effect, the $\mathrm{X}$-ray source was disabled and the pulse frequency was varied between $10 \mathrm{kHz}$ and $200 \mathrm{kHz}$. With increased pulse frequency an increased detector current could be measured. This current is caused by a parasitic capacitive effect due to the design with parallel ring electrodes leading to a significant displacement current. This displacement current is directly opposite to the electron current, thus superimposing the measurement signal.

Therefore, in order to reduce this displacement current, the design was modified to resemble the classical radioactive ECD as shown in Fig. 3. Therefore, the

\footnotetext{
${ }^{1} \mathrm{ml} / \mathrm{min}$ (milliliter standard per minute): mass flow at reference conditions $20^{\circ} \mathrm{C}$ and 1013 mbar.
} 
analyte inlet capillary is connected to the transimpedance amplifier and thus used as the detector electrode. The rings 1-3 are short-circuited and connected to the voltage $U$ as the pusher electrode.

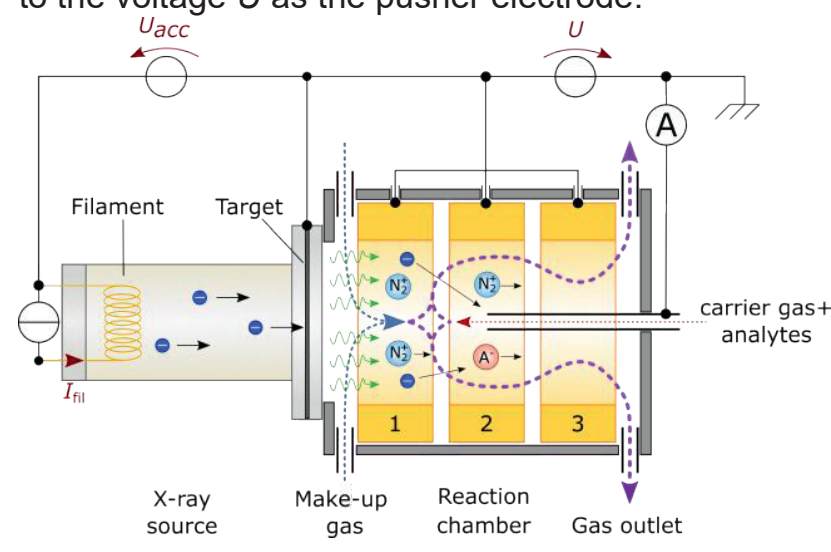

Fig. 3: Modified reaction chamber of the X-ray based ECD with the analyte inlet capillary as the detector electrode.

Using the shown structure, the displacement current is reduced to a negligible value and thus the electron current is measurable.

Now, the optimum pulse width and amplitude of $U$ using this improved setup were determined as described above to $U=-15 \mathrm{~V}$ with a pulse width of $0.5 \mu \mathrm{s}$. These values are used in the following. The resulting frequency change depending on the 1,1,2-TCE concentration is measured and shown in Fig. 4 (orange dots).

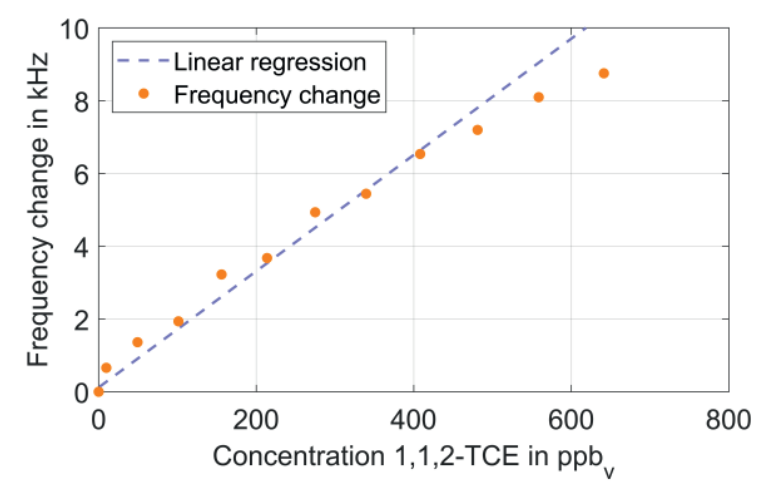

Fig. 4: Frequency change depending on the 1,1,2-trichloroethane concentration using the X-ray ECD setup from

Fig. 3.

The linear regression for lower concentrations is shown as blue dashed line. Using this regression, a detection limit of about $4.5 \mathrm{ppb}_{\mathrm{v}}$ at an averaging time of $200 \mathrm{~ms}$ is reached for 1,1,2-TCE, which is comparable to the detection limit of radioactive ECDs for 1,1,2-TCE [11]. Furthermore, an approximately linear behavior can be seen over a range from the detection limit of $4.5 \mathrm{ppb}_{\mathrm{v}}$ to a concentration of about $480 \mathrm{ppb}_{\mathrm{v}}$.
This just results in a limited linear dynamic range of about $10^{2}$.

\section{Conclusions}

In this work we present preliminary results of an ECD based on X-rays. A commercially available $X$ ray source was chosen and operated at low accelerating voltage of less than $5 \mathrm{kV}$ avoiding costly regulatory requirements during operation. A detection limit of about $4.5 \mathrm{ppb}_{\mathrm{v}}$ at an averaging time of $200 \mathrm{~ms}$ is reached for 1,1,2-trichloroethane, which is comparable to the detection limit of radioactive ECDs for 1,1,2-trichloroethane [11]. In terms of linearity, a linear dynamic range of $10^{2}$ from $4.5 \mathrm{ppb}_{\mathrm{v}}$ to $480 \mathrm{ppb}_{\mathrm{v}}$ has been achieved which in comparison to radioactive ECDs is less by two orders of magnitude. Increasing the linear dynamic range is part of ongoing research.

\section{References}

[1] J.E. Lovelock, A sensitive detector for gas chromatography, J. Chrom. A 1 (1958) 35-46.

[2] J.E. Lovelock, S.R. Lipsky, Electron Affinity Spectroscopy-A New Method for the Identification of Functional Groups in Chemical Compounds Separated by Gas Chromatography 1, J. Am. Chem. Soc. 82 (1960) 431-433.

[3] E. Bunert, B. Bernhold, J.N. Woidtke, M. SehImeyer, S. Zimmermann, Non-radioactive electron capture detector for gas chromatography - A possible replacement for radioactive detectors, Journal of chromatography. A 1606 (2019) 460384.

[4] R.J. Maggs, P.L. Joynes, A.J. Davies, J.E. Lovelock, Electron capture detector. New mode of operation, Anal. Chem. 43 (1971) 1966-1971.

[5] W.E. Wentworth, E.D. D'Sa, H. Cai, S. Stearns, Environmental Applications of the Pulsed-Discharge Electron-Capture Detector, J. Chromatogr. Sci. 30 (1992) 478-485.

[6] A. Morozov, T. Heindl, C. Skrobol, J. Wieser, R. Krücken, A. Ulrich, Transmission of $\sim 10 \mathrm{keV}$ electron beams through thin ceramic foils: Measurements and Monte Carlo simulations of electron energy distribution functions, Eur. Phys. J. D 48 (2008) 383-388.

[7] M. Sámel, M. Stano, M. Zahoran, M. Ries, Š. Matejčík, Experimental characterisation of atmospheric pressure electron gun, Int. J. Mass Spectrom. 439 (2019) 34-41.

[8] W. Bothe, Röntgenstrahlung Ausschliesslich Röntgenoptik: Band XXIII · Zweiter Teil, Springer, Berlin, Heidelberg, 1933.

[9] I.V. Hertel, C.-P. Schulz, Atome und Grundlagen ihrer Spektroskopie, 2nd ed., Springer Spektrum, Berlin, 2017.

[10] P. Cochems, A.T. Kirk, S. Zimmermann, In-circuitmeasurement of parasitic elements in high gain high bandwidth low noise transimpedance amplifiers, Rev. Sci. Instrum. 85 (2014) 124703. 
[11] F. Barani, N. Dell'Amico, L. Griffone, M. Santoro, C. Tarabella, Determination of Volatile Organic Compounds by Headspace Trap, Journal of Chromatographic Science 44 (2006) 625-630. 